\title{
Tunable Plasmon Resonance in Ag-Au Split-Ring Resonator with Inner Metallic Cross Heterogeneous Dimer Array
}

\author{
Xin Zhou, ${ }^{1,2}$ Shuangchun Wen, ${ }^{1}$ Bin Tang, ${ }^{3}$ Diwu Yang, ${ }^{2}$ and Jun $\mathrm{He}^{2}$ \\ ${ }^{1}$ Key Laboratory for Micro-Nano-Optoelectronic Devices of Ministry of Education, College of Physics and Microelectronic Science, \\ Hunan University, Changsha 410082, China \\ ${ }^{2}$ School of Sciences, Hunan University of Technology, Zhuzhou 412008, China \\ ${ }^{3}$ School of Mathematics \& Physics, Changzhou University, Changzhou 213164, China
}

Correspondence should be addressed to Shuangchun Wen; scwen@hnu.edu.cn

Received 16 July 2015; Accepted 14 September 2015

Academic Editor: Sabine Szunerits

Copyright (C) 2015 Xin Zhou et al. This is an open access article distributed under the Creative Commons Attribution License, which permits unrestricted use, distribution, and reproduction in any medium, provided the original work is properly cited.

The plasmon resonance behavior in Ag-Au split-ring resonator (SRR) heterogeneous dimer array has been investigated numerically by using the finite difference time domain (FDTD) method. The resonance dips were tuned with the metallic SRR dimer arrays constituted by different metals. For the Ag-Au heterogeneous SRRs dimer array, with the arms of metallic cross lengths increasing, the resonance dips in the visible region red shifted but the locations of resonance modes in the near-infrared region kept immovability. On the contrary, the locations of resonance dips in the visible region kept immovability but the resonance modes in the near-infrared region red shifted obviously with the air cuts widths decreasing. The whole resonance dips exhibited a red-shift as the environmental dielectric constants increase. The electric and magnetic field distributions of certain resonance wavelengths were also investigated.

\section{Introduction}

In recent years, metamaterials researches have attracted an enormous amount of attention [1-3]. Metamaterials are fascinating artificial materials which have a special advantage to control electromagnetic waves propagation and especially adjust the light polarization state through different designs or diverse spatial arrangements of structural unit. Metallic splitring resonators (SRRs) are the most outstanding examples for metamaterial building block $[4,5]$. By combining this artificial atoms array with an array of nanorods, in microwave regime, Smith et al. [6] experimentally revealed the metamaterials possess negative refractive index. Since then, metallic SRRs have been developed rapidly in shifting the resonant response to higher frequencies [7-9]. Due to the unique nature of SRRs, there are massive studies in the literature with regard to the multifarious aspects of SRRs. Some researches have reported the numerical simulation results of the effective parameters $[10,11]$ and the transmission characteristics [12] of SRR structures. Transmission properties of disordered and periodic SRRs [13] have been discussed experimentally at length. Electromagnetic resonance features of SRRs have been considered widely in both experimental and theoretical methods [14]. The influence of structure parameters on SRRs' resonance behaviours and the origin of resonances have been analyzed in detail [15].

However, the left-handed metamaterials composed of SRRs are very sensitive to the polarization of the incident electromagnetic field. To solve the problem, Padilla et al. [16] designed a metamaterial built up of pairs of metallic crosses, which are similar to the rosette design for artificial gyrotropic devices and metamaterials. These wires act as small electrical dipoles which lead to a negative effective permittivity [17]. Additionally, two opposing crossbars within one elementary cell form some kind of $L C$-resonance circuit, which can be excited by a magnetic flux through the area between the two wires [18]. Then, Chen et al. [19] presented the results of experimental and computational studies on different 
planar SRR with inner metallic cross metamaterials. These metamaterial structures and their corresponding inverse structures showed complementary transmission properties as characterized by THz time domain spectroscopy. Since then, homologous metamaterial structures have been studied by some researchers $[20,21]$.

As we all know, the surface plasmon polaritons (SPPs) are coupled oscillations of electromagnetic fields and electrons [22]. Recently, plasmonic metallic nanostructures have obtained massive interest owing to their potential applications in biological or chemical sensing [23], biomedicine [24], surface enhanced spectroscopy [25], optoelectronics [26], optical filters and sensors [27], and other areas. Plasmon hybridizations and interferences in the complex metallic nanostructures provide a powerful means to tailor the spectral response and reinforce local field at nanometer scale. It has been demonstrated that the nanoparticle dimer has strong local electromagnetic field enhancements in the dimer gaps [28]. The metallic dimer nanostructures can exhibit a large number of attractive effects, such as radiation damping modification, optical frequency mixing, and plasmonic Fano resonances [29]. SPPs resonance characteristics of metallic dimer nanostructures are highly dependent on the metal composition, size, shape, periodicity of its elements, and surrounding environment. The optical response of $\mathrm{Au}-\mathrm{Ag}$ plasmonic heterodimers has been previously studied theoretically and experimentally [30].

Motivated by the above researches, in this paper, we proposed the Ag-Au split-ring resonator with inner metallic cross heterogeneous dimer array with different metal compositions, arms of metallic cross lengths, widths of air cuts, and environmental dielectric constants. The simulated numerical results showed that the resonance modes can be tuned by different metal compositions of the SRR dimer arrays. For the Ag-Au heterogeneous SRRs dimer array, along with the arms of metallic cross lengths increasing, the resonance dips in the visible region red shifted but the locations of resonance modes in the near-infrared region kept immovability. On the contrary, the locations of resonance dips in the visible region kept immovability but the resonance modes in the nearinfrared region red shifted obviously with the air cuts widths decreasing. With the environmental dielectric constants $\varepsilon$ increasing, the whole resonance dips presented a red-shift. Moreover, the electric and magnetic field distributions of certain resonance wavelengths were also investigated.

\section{Structure and Simulation Method}

The schematic view of a unit cell of the SRR with inner metallic cross dimmer array was given in Figure 1. The grey and yellow background materials indicated the metal silver and gold, respectively. The nanostructure we proposed was immersed in a vacuum. All the split rings had the same width $t=$ $10 \mathrm{~nm}$ and outer radius $R=50 \mathrm{~nm}$. Additionally, the edgeto-edge spacing between adjacent split rings and the arm of the metallic cross width were set to be $d=20 \mathrm{~nm}$ and $t=10 \mathrm{~nm}$, respectively. In this work, the variable parameters are the following: the arm of metallic cross length $l$, the width of each air cut $w$, and the permittivity of medium in the two

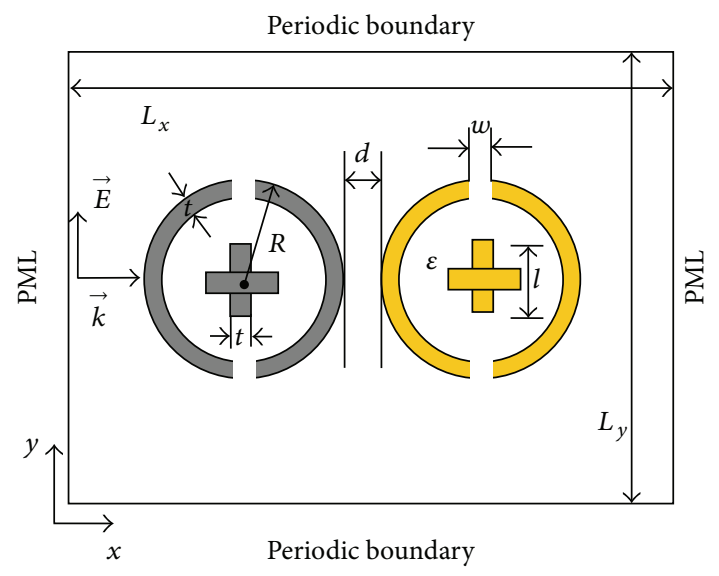

FIGURE 1: $x-y$ cross section of a unit cell of the Ag-Au split-ring resonator with inner metallic cross heterogeneous dimer array. Parameters are defined in the text.

split rings $\varepsilon$. The frequency-dependent dielectric constants $\varepsilon_{m}$ of gold and silver are given approximated by the Drude model as $[31,32]$

$$
\varepsilon_{m}(\omega)=\varepsilon_{\infty}-\frac{\omega_{p}^{2}}{\omega^{2}+i \omega \gamma_{p}},
$$

where $\varepsilon_{\infty}$ expresses the instantaneous relative dielectric constant at the infinite frequency, $\omega_{p}$ denotes the bulk plasma frequency of metal, $\omega$ is the angle frequency of the incident wave, and $\gamma_{p}$ represents the collision frequency, respectively. The dielectric constants of both metals are taken from [33, 34].

The time-dependent near field and transmission spectra of the SRR dimer structure were calculated by using twodimensional finite-difference time domain (FDTD) method [35]. As depicted in Figure 1, the proposed SRR dimer array was illuminated by a normal incident Gaussian single pulse wave with propagation vector $(\vec{k})$ along the $x$-direction and electric field vector $(\vec{E})$ along the $y$-direction. The analyzed structure was simulated by an FDTD cube of size $L_{x} \times L_{y}=$ $800 \mathrm{~nm} \times 400 \mathrm{~nm}$ and infinity along the $z$-axis. The spatial and temporal steps were set as $\Delta x=\Delta y=1 \mathrm{~nm}$ and $\Delta t=\Delta x / 2 c$ ( $c$ is the speed of light in vacuum). Also, perfectly matched layer (PML) absorbing boundary condition and periodic boundary condition were applied along the $x$ - and $y$-axes, respectively.

\section{Results and Discussion}

3.1. Plasmon Resonance in SRR Arrays Constituted by Different Metals. To start with, Figure 2 depicts the transmission spectra for three SRRs with inner cross dimer arrays which constituted $\mathrm{Au}$ (red line), Ag (blue line), and Ag-Au (black line) for comparison. The three nanostructures had same structure parameters $(\varepsilon=1, l=25 \mathrm{~nm}$, and $w=10 \mathrm{~nm})$ except for different metal compositions. The first thing we noted was that three transmission spectra exhibited the same plasmon resonance peak at wavelength $\lambda=0.804 \mu \mathrm{m}$ and the peak intensity achieved 1.0. In the later paragraphs, we will see that the location of this resonance peak still 


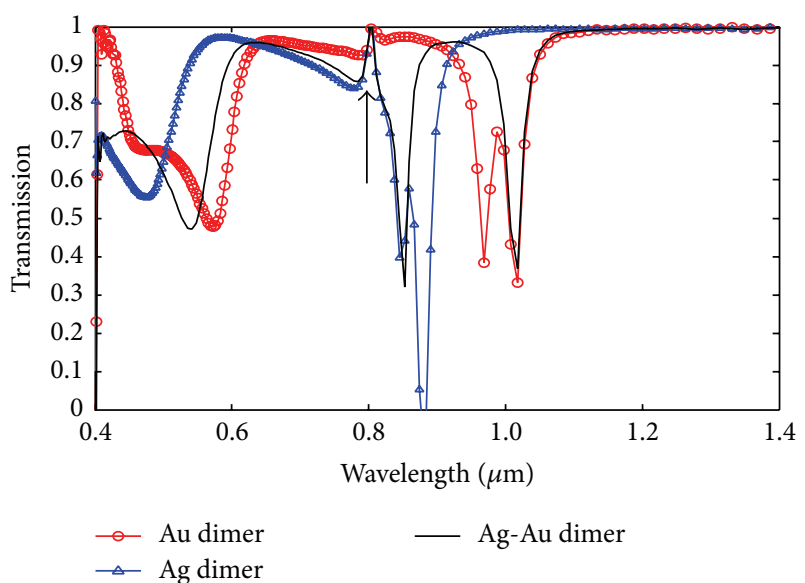

Figure 2: Transmission spectra for Au dimer array (red line), Ag (blue line) dimer array, and Ag-Au heterogeneous dimer array (black line). The width of air cut, a nanorod of metallic cross length, and the permittivity of medium in the rings in all the structures are set to be $w=10 \mathrm{~nm}, l=25 \mathrm{~nm}$, and $\varepsilon=1$.

keeps fixedness with different structure parameters of the nanosystem. Therefore, the formation of this plasmon resonance peak is independent of material components and structure parameters of SRR dimer arrays. It may originate from periodicity of structure. In the visible region, all the spectra had a dip which possessed different intensities and positions. For example, Ag-Au SRR heterogeneous dimer array had a dip located at $\lambda=0.5386 \mu \mathrm{m}$ with intensity 0.471 , whereas Ag homogenous dimer array represented the dip located at $\lambda=0.4744 \mu \mathrm{m}$ with intensity 0.555 . The SPPs resonance wavelength is related to the permittivity of the medium and the metals $\varepsilon_{m}$ and $\varepsilon_{d}$. In the case of same permittivity $\varepsilon_{d}=1$, there is $\operatorname{Re}\left|\varepsilon_{m}(\mathrm{Au})\right|<\operatorname{Re}\left|\varepsilon_{m}(\mathrm{Ag})\right|$ of the permittivity of the $\mathrm{Ag}$ and $\mathrm{Au}$ at this wavelength range, which leads to the difference between the locations of resonance dips for three types of structure. At the wavelengths greater than $\lambda=1.8 \mu \mathrm{m}$ region, all the spectra exhibited $100 \%$ transmission efficiency. In addition, the transmission spectra of the $\mathrm{Au}$ and $\mathrm{Ag}$ homogenous dimer arrays showed an acute peak in the near-infrared region, which located at wavelength $\lambda=0.9876 \mu \mathrm{m}$ and $\lambda=0.8596 \mu \mathrm{m}$, respectively. On the contrary, a relative wider resonance peak appeared in the wavelength region $\lambda=0.8522 \mu \mathrm{m}$ to $\lambda=1.018 \mu \mathrm{m}$ in the spectra of the Ag-Au heterogeneous dimer array. Different materials can be used to adjust the SPPs resonances properties of the nanoparticles. By combining the advantages of different materials, the composite nanostructure can realize the electromagnetic coupling between metal nanoparticles and adjust the plasmon resonances. The plasmon resonant behaviors of the three nanostructures are influenced by different dispersion of Ag and Au.

To further understand more basic physical mechanisms and information of the resonance peaks, the spatial distributions of the electric and magnetic were also given. Figure 3 exhibits the calculated field components $E_{x}$ and $H_{z}$ distributions at the same resonance wavelength $\lambda=0.804 \mu \mathrm{m}$ (as shown by the up arrow in Figure 2) for three different nanostructures: ( $\mathrm{a}$ and b) Au homogenous dimer array, (c and d) $\mathrm{Ag}$ homogenous dimer array, and (e and f) $\mathrm{Ag}-\mathrm{Au}$ heterogeneous dimer array. According to the boundary condition of $\vec{n} \cdot \vec{E}=\sigma / \varepsilon_{0}$ and $\vec{n}=n \vec{x}$ in the normal direction, we know that $E_{x}$ distributions are associated with surface charge densities $\sigma$. Figures 3(a), 3(c), and 3(e) all indicated massive surface charge densities accumulated in the air cuts of the split rings. Meanwhile, positive and negative $E_{x}$ fields distributed (shown by red and blue areas) in the two air cuts of every ring were just opposite. For Au homogenous dimer array, quadrupole modes were found outside the two rings and dipole mode formed in the left ring. A weak quadrupole mode was also found at the corners of Au cross in the right ring, which showed a typical edge effect. For Ag homogenous dimer array, dipole modes formed in the two rings. The electric field between the two split rings was strengthened. For Ag-Au heterogeneous dimer array, the electric field between the two split rings was further strengthened. The left Ag split ring and the right Au split ring exhibited the same $E_{x}$ field distributions as the left ring in Figure 3(c) and as the right ring in Figure 3(a), respectively. But the electric field was strengthened, which could be due to the stronger coupling of SPPs excited from $\mathrm{Ag}$ and $\mathrm{Au}$, as the dispersion of $\mathrm{Ag}$ and $\mathrm{Au}$ is different.

Then, we discussed the magnetic field component $H_{z}$ distributions which were shown in Figures 3(b), 3(d), and 3(f). For Au homogenous dimer array, the weak positive magnetic fields were fairly well-distributed in the two rings. On the contrary, the stronger negative $H_{z}$ field distributions were found in the two split rings in Figure 3(d), but the field in the right split ring was more stronger than that in the left ring. In Figure 3(f), the left $\mathrm{Ag}$ split ring and the right $\mathrm{Au}$ split ring showed the negative and positive $H_{z}$ fields, the same as $\mathrm{H}_{z}$ field distributed in the left and right rings in Figures 3(d) and $3(\mathrm{~b})$, respectively.

\subsection{Plasmon Resonance in Ag-Au SRR Heterogeneous Dimer} Array. In this section, we reported detailed numerical results of the Ag-Au SRR heterogeneous dimer array to demonstrate the plasmon resonance characteristics. For this purpose, we compared the transmission behaviors for Ag-Au SRR heterogeneous dimer arrays with different arm of metallic cross lengths $l$, widths of each air cut $w$, and permittivities of medium in the two split rings $\varepsilon$.

As shown in Figure 4, the permittivity of medium in the two split rings $\varepsilon=1$ and the widths of each air cut $w=10 \mathrm{~nm}$ were fixed, along with the arms of metallic cross lengths $l$ increasing from $25 \mathrm{~nm}$ to $40 \mathrm{~nm}$, and the resonance dips at the $\lambda=0.8522 \mu \mathrm{m}$ and $\lambda=1.018 \mu \mathrm{m}$ were further deepened. At the same time, the resonance dips in the visible region red shifted but the locations of resonance modes in the near-infrared region kept immovability (as shown by dash lines in Figure 4). For instance, the dip in the visible region decreased from a $47 \%$ transmittance at $\lambda=0.539 \mu \mathrm{m}$ for $l=25 \mathrm{~nm}$ to a $42 \%$ transmittance at $\lambda=0.583 \mu \mathrm{m}$ for $l=$ $40 \mathrm{~nm}$. This indicates that the cross size mainly influences the resonance mode in the visible region but it is unrelated to the resonance modes in the near-infrared region. The decrease of 


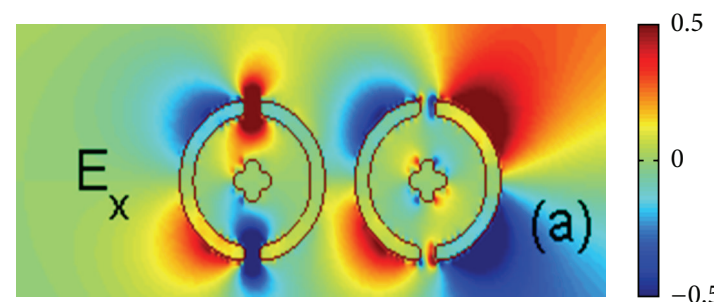

(a)

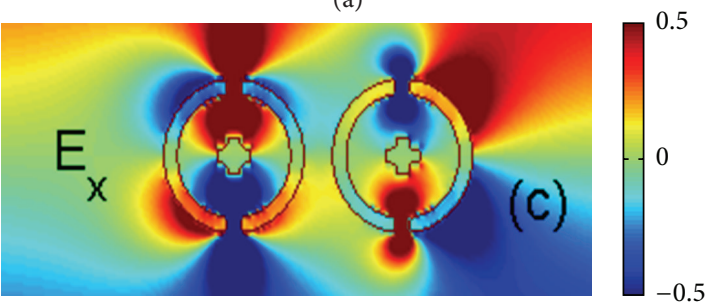

(c)

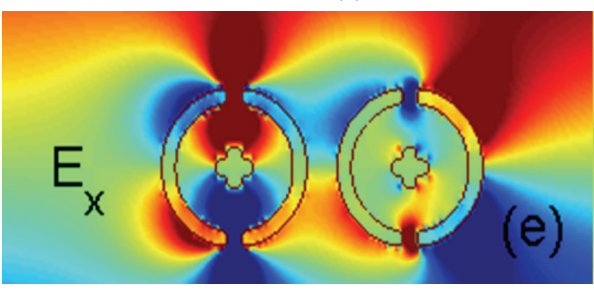

(e)

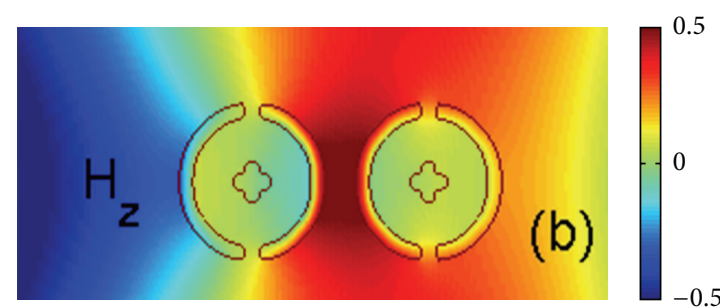

(b)

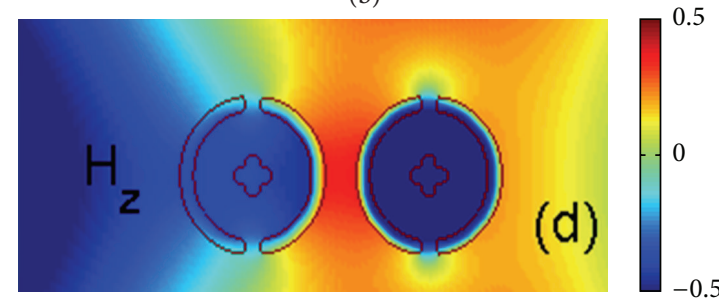

(d)

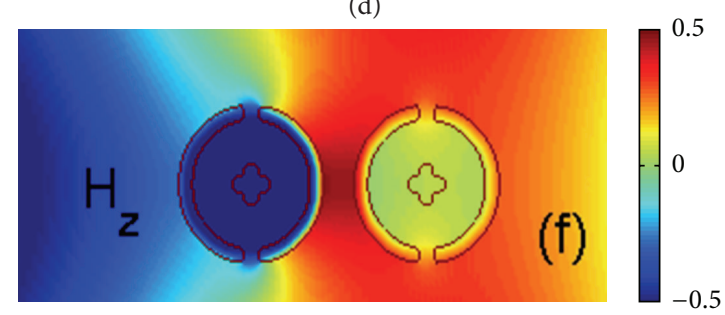

(f)

Figure 3: The electric and magnetic field components $E_{x}$ and $H_{z}$ distributions at the same resonance wavelength $\lambda=0.804 \mu \mathrm{m}$ (as shown by the up arrow in Figure 2) for three different nanostructures: ( $a$ and b) Au homogenous dimer array, ( $c$ and d) Ag homogenous dimer array, and (e and f) Ag-Au heterogeneous dimer array.

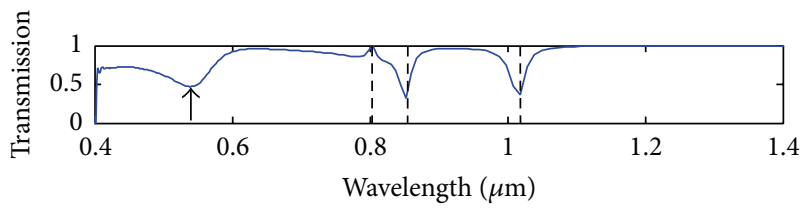

(a) $l=25 \mathrm{~nm}$

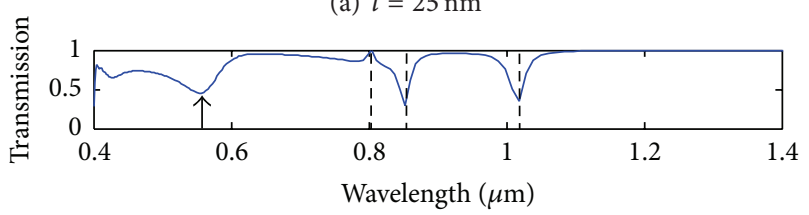

(b) $l=30 \mathrm{~nm}$

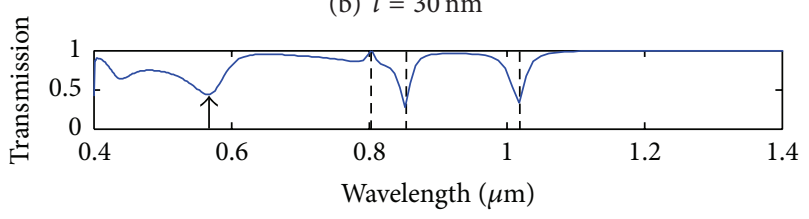

(c) $l=35 \mathrm{~nm}$

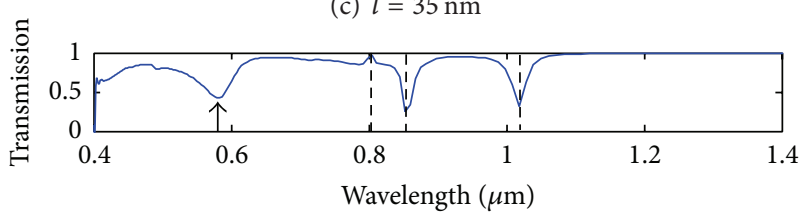

(d) $l=40 \mathrm{~nm}$

FIgURE 4: Transmission spectra of the Ag-Au SRR heterogeneous dimer arrays as a function of wavelength for different nanorods of metallic cross lengths $l$. transmission efficiency for the resonance dips with increasing $l$ is due to the introduction of more metal gold to increase the absorption and reflection for the incident light.

In order to understand the causes of the changes for the dips in the visible region, we calculated the electric field components $E_{x}$ and $E_{y}$ distributions for the resonance dips indicated by the up arrows in Figure 4 . The corresponding results were presented in Figure 5, and the four resonance dips located at (a-b) $0.539 \mu \mathrm{m}$, (c-d) $0.554 \mu \mathrm{m},(d-f) 0.569 \mu \mathrm{m}$, and (g-h) $0.583 \mu \mathrm{m}$, respectively. Firstly, in Figure 5(a), the quadrupole modes distributed outside the two split rings and dipole modes formed in the air cuts. It is worthwhile to note that the same electric field $E_{x}$ distributions outside the rings and in the air cuts were found in Figures 5(c), 5(e), and 5(g). Secondly, quadrupole modes existed at the edge of metallic cross, as shown in Figures 5(a), 5(c), and 5(e), and the electric field gradually was intensified. In particular, Figure 5(g) showed multipole plasmon modes that appeared at the edges of Ag cross in the left Ag split ring, but quadrupole mode still distributed in the right Au split ring. Finally, as shown in Figures 5(b), 5(d), 5(f), and 5(h), all the plots exhibited the same electric field $E_{y}$ distributions. Positive and negative $E_{y}$ fields distributed in the left Ag ring and right Au ring, respectively. Furthermore, quadrupole modes appeared outside all the split rings. After synthesizing the above phenomena, we can know that increasing $l$ almost has no influence on the outside of the split rings. The changes of resonance dips in 


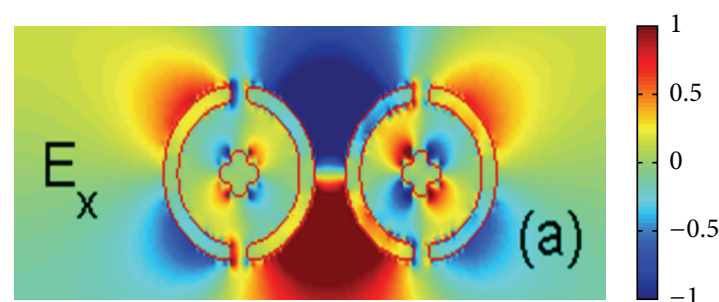

(a)

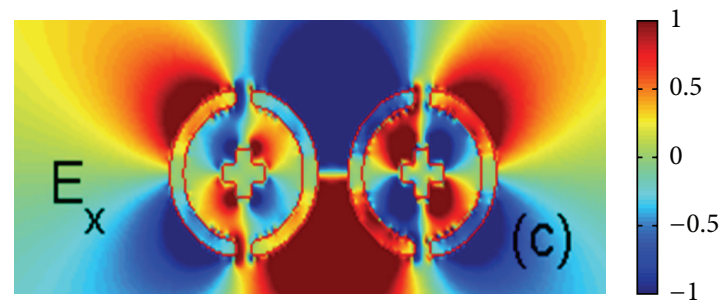

(c)

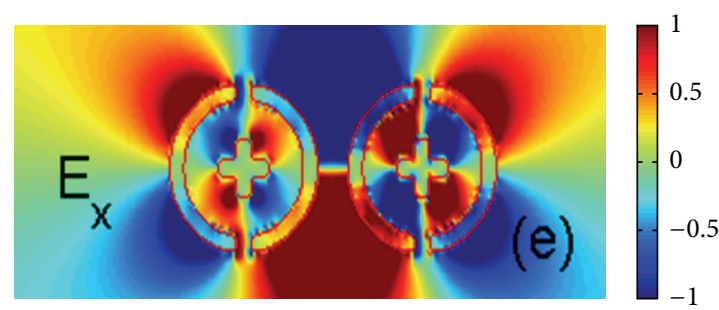

(e)

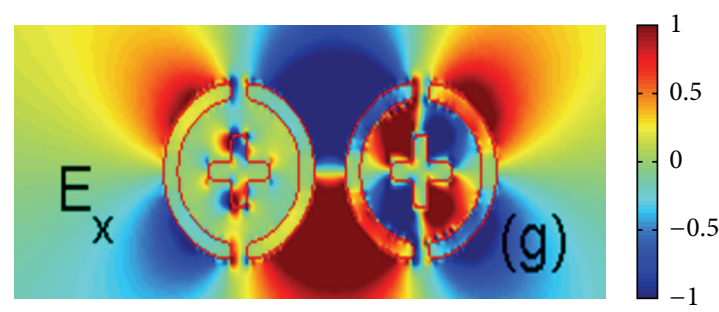

$(\mathrm{g})$

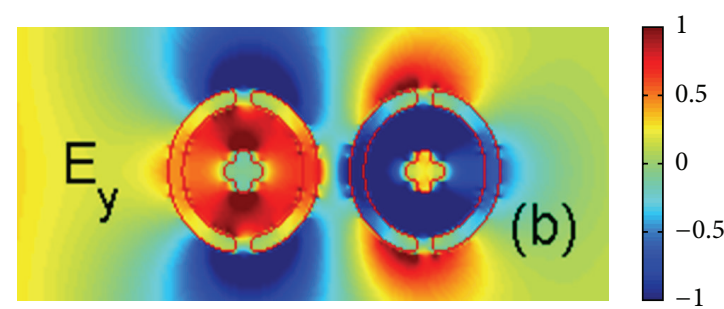

(b)

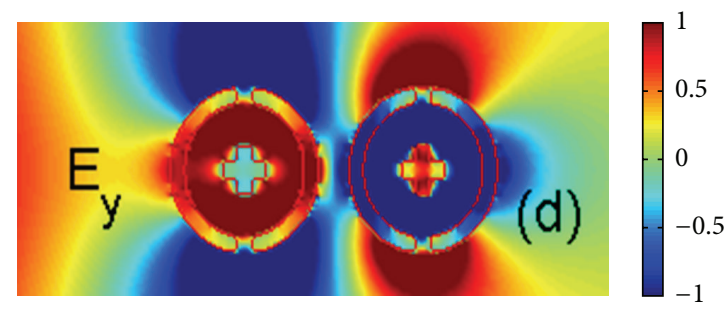

(d)

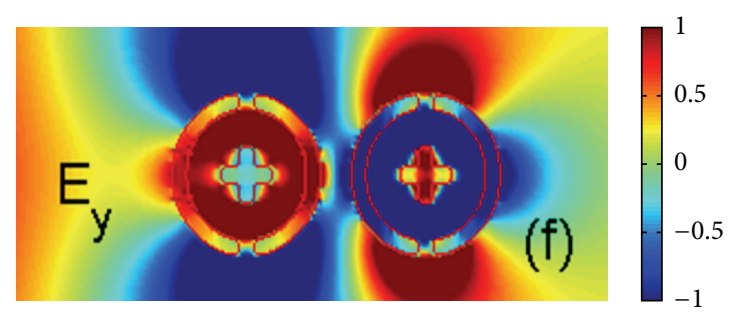

(f)

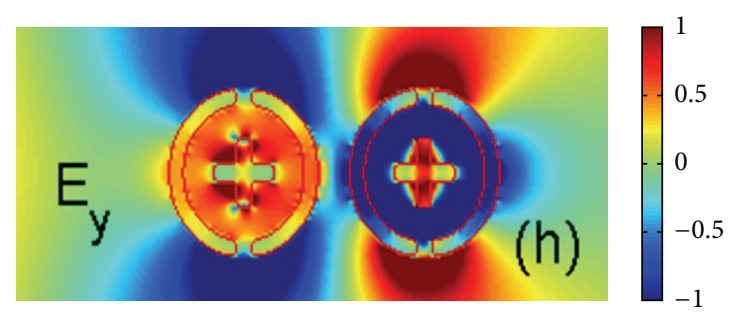

(h)

Figure 5: The electric field components $E_{x}$ and $E_{y}$ distributions at four different resonance dips shown with the up arrows in Figure 4, and the resonance dips are located at (a-b) $0.539 \mu \mathrm{m},(\mathrm{c}-\mathrm{d}) 0.554 \mu \mathrm{m},(\mathrm{d}-\mathrm{f}) 0.569 \mu \mathrm{m}$, and (g-h) $0.583 \mu \mathrm{m}$, respectively.

the visible region were only associated with the electric field around the metallic cross.

Next, we kept $l=25 \mathrm{~nm}$ and $\varepsilon=1$ as constants while the air cuts widths $w$ were varied from $12 \mathrm{~nm}$ to $6 \mathrm{~nm}$. Figure 6 shows the corresponding simulated transmission spectra. Contrary to Figure 4, the locations of resonance dips in the visible region kept immovability (as shown by the dash line in Figure 6) but the resonance modes in the near-infrared region red shifted obviously. A comparison of Figures 4 and 6 indicates that the location of the resonance modes in the near-infrared region is mainly determined by the parameters of the split rings, but the location of the resonance dip in the visible region is mainly determined by the parameters of the metallic cross. Furthermore, the two resonance dips in the near-infrared region moved farther apart with decreasing $w$. The transmission efficiency was gradually increased on both sides of the resonance peak located at $\lambda=0.804 \mu \mathrm{m}$, so the resonance peak disappeared eventually. Due to the changes of the transmission spectra mentioned above, there emerged two wider transparent windows between the wavelength region $\lambda=0.6 \mu \mathrm{m}$ to $\lambda=0.9 \mu \mathrm{m}$ and the region $\lambda=1.0 \mu \mathrm{m}$ to $\lambda=1.3 \mu \mathrm{m}$ when $w=6 \mathrm{~nm}$ (as shown in Figure 6(d)).

The split-ring resonator with inner metallic cross still can be described as an equivalent $L C$ circuit, in which the air cuts and the metallic ring represent equivalent capacitance $(C)$ and effective inductance $(L)$, respectively $[36,37]$. Here, the equivalent capacitance $C$ for the accumulated charge in the air cuts depends on the geometry of the cuts. The effective 


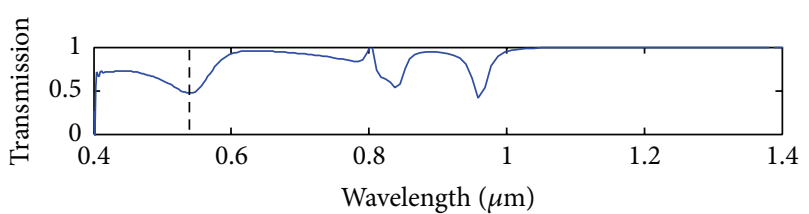

(a) $w=12 \mathrm{~nm}$

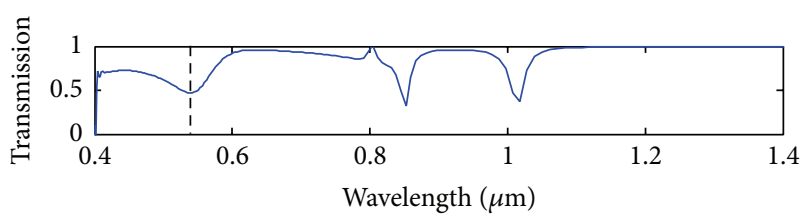

(b) $w=10 \mathrm{~nm}$

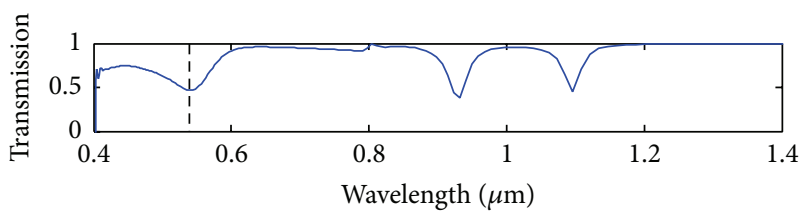

(c) $w=8 \mathrm{~nm}$

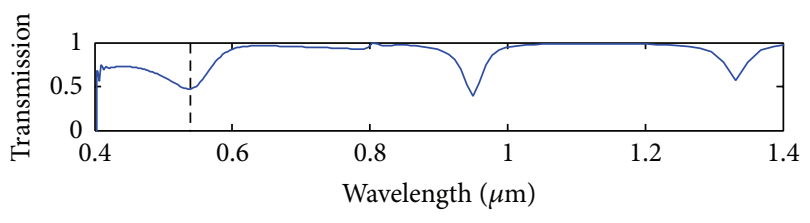

(d) $w=6 \mathrm{~nm}$

Figure 6: Transmission spectra of the Ag-Au SRR heterogeneous dimer arrays as a function of wavelength for different air cuts widths $w$.

inductance $L$ is influenced by the geometry of the metallic rings [38]. The corresponding resonant frequency can be written as

$$
\begin{aligned}
& \omega=\frac{1}{\sqrt{L C}}, \\
& C \propto \frac{\varepsilon S}{d},
\end{aligned}
$$

where $\varepsilon$ expresses the permittivity, $S$ shows the area of the capacitor, and $d$ denotes the distance of the cuts. The air cuts behave like parallel plate capacitors. We can consider two metal plates with width $10 \mathrm{~nm}$ that are placed with a distance $w$ between them. When the cuts widths decrease, the capacitance due to air cuts will increase. Consequently, the total capacitance of the nanosystem will increase, which in turn will decrease the resonant frequency according to (2). So a red-shift occurred in the resonance dips in the near-infrared region. Previously, [39, 40] have predicted and verified the same behavior: increasing the capacitance of the nanosystem will increase the resonance wavelength.

The resonance frequency of the Ag-Au SRR with inner metallic cross heterogeneous dimer array also depends on the permittivity of the surrounding material. Figure 7 presents the transmission spectra as the permittivities of medium in the two split rings $\varepsilon$ were varied from 1 to 2.5 in steps of 0.5 , while the other parameters $l=25 \mathrm{~nm}$ and $w=10 \mathrm{~nm}$ kept constants. Similar to Figure 5, the transmission efficiency

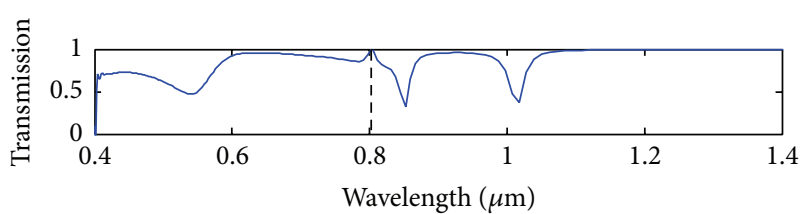

(a) $\varepsilon=1$

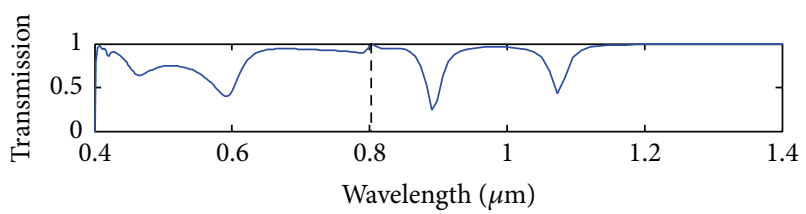

(b) $\varepsilon=1.5$

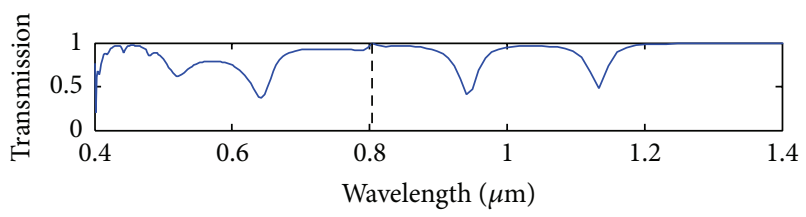

(c) $\varepsilon=2$

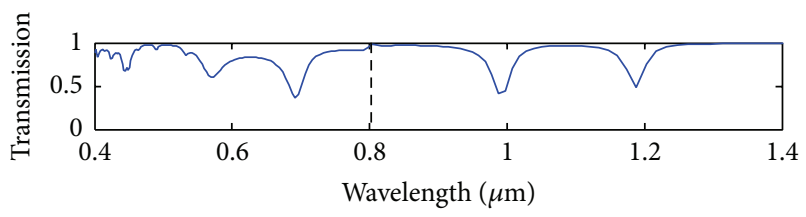

(d) $\varepsilon=2.5$

FIgURE 7: Transmission spectra of the Ag-Au SRR heterogeneous dimer arrays as a function of wavelength for different environmental dielectric constants $\varepsilon$.

was also gradually increased on both sides of the resonance peak located at $\lambda=0.804 \mu \mathrm{m}$ and the location of the peak kept fixedness (as shown by the dash line in Figure 7). The whole resonance dips exhibited a red-shift and there appeared more and more irregular dips in visible wavelengths range with increasing environmental dielectric constant $\varepsilon$. There are two reasons for the red-shift of the transmission spectra. On the one hand, on the basis of (3), as we increase the permittivity from 1 to 2.5 , capacitance $C$ increases. As a result, the resonant frequency decreases. On the other hand, the SPP resonances can be considered as an electron gas oscillating. The restoring force is provided by induced surface charges. When the medium fills in the split rings, it polarizes and thus reduces the surface charges strength. Therefore, restoring force is decreased and the plasmon energies are lowered. In addition, the polarization effects become stronger with the increment of dielectric constant value. The combined effects of above two factors result in a red-shift of the transmission spectra.

\section{Conclusions}

In summary, we have investigated numerically the plasmon resonance behavior in Ag-Au SRR heterogeneous dimer array by using $2 \mathrm{D}$ FDTD method. In $[17,18]$, the authors utilized different semi-insulating substrates (GaAs or SI-GaAs) to control the electric response properties of SRRs. It is usually 
considered that the carrier concentration of the substrate mainly affects the size and property of the gap in the SRRs, which control the $L C$ resonant response of the nanostructure. Different from the above references, the interest of this study was the plasmon resonance properties of the heterogeneous SRRs dimer array without substrate. It was found that the resonance dips were changed with the metallic SRR with inner metallic cross arrays constituted by different metals. For the Ag-Au heterogeneous SRRs dimer array, different resonance modes could tune by the following structure parameters: arm of metallic cross length $l$, the width of each air cut $w$, and the permittivity of medium in the two split rings $\varepsilon$. The electric and magnetic field distributions of certain resonance wavelengths have been given to further understand more basic physical mechanisms and information of the resonance peaks and dips. The proposed nanostructure potentially allows heterogeneous plasmonic dimer to be used in new optical device.

\section{Conflict of Interests}

The authors declare that there is no conflict of interests regarding the publication of this paper.

\section{Acknowledgments}

This work was funded by Hunan provincial Nature Science Foundation of China (Grant no. 14JJ3122), Scientific Research Foundation for Excellent Youth of Hunan Provincial Education Department (Grant no. 15B065), Natural Science Foundation of Jiangsu Province of China (Grant no. BK20141169), China Postdoctoral Science Foundation (Grant no. 2015M570674), and the National Natural Science Foundation of China (Grant nos. 11374094 and 61401153).

\section{References}

[1] V. A. Fedotov, M. Rose, S. L. Prosvirnin, N. Papasimakis, and N. I. Zheludev, "Sharp trapped-mode resonances in planar metamaterials with a broken structural symmetry," Physical Review Letters, vol. 99, no. 14, Article ID 147401, 2007.

[2] Y. Cui, L. Kang, S. Lan, S. Rodrigues, and W. Cai, "Giant chiral optical response from a twisted-arc metamaterial," Nano Letters, vol. 14, no. 2, pp. 1021-1025, 2014.

[3] X. Shen and T. J. Cui, "Ultrathin plasmonic metamaterial for spoof localized surface plasmons," Laser and Photonics Reviews, vol. 8, no. 1, pp. 137-145, 2014.

[4] P. Ding, E. J. Liang, W. Q. Hu, G. W. Cai, and Q. Z. Xue, “Tunable plasmonic properties and giant field enhancement in asymmetric double split ring arrays," Photonics and Nanostructures: Fundamentals and Applications, vol. 9, no. 1, pp. 42-48, 2011.

[5] S. Linden, F. B. P. Niesler, J. Förstner, Y. Grynko, T. Meier, and M. Wegener, "Collective effects in second-harmonic generation from split-ring-resonator arrays," Physical Review Letters, vol. 109, no. 1, Article ID 015502, 2012.

[6] D. R. Smith, W. J. Padilla, D. C. Vier, S. C. Nemat-Nasser, and S. Schultz, "Composite medium with simultaneously negative permeability and permittivity," Physical Review Letters, vol. 84, no. 18, Article ID 4184, 2000.
[7] J. Pan, Z. Chen, Z.-D. Yan et al., "Symmetric and anti-symmetric magnetic resonances in double-triangle nanoparticle arrays fabricated via angle-resolved nanosphere lithography," AIP Advances, vol. 1, no. 4, Article ID 042114, 2011.

[8] C. Enkrich, M. Wegener, S. Linden et al., "Magnetic metamaterials at telecommunication and visible frequencies," Physical Review Letters, vol. 95, no. 20, Article ID 203901, 2005.

[9] T. Okamoto, T. Otsuka, S. Sato, T. Fukuta, and M. Haraguchi, "Dependence of LC resonance wavelength on size of silver splitring resonator fabricated by nanosphere lithography," Optics Express, vol. 20, no. 21, pp. 24059-24067, 2012.

[10] M. Decker, N. Feth, C. M. Soukoulis, S. Linden, and M. Wegener, "Retarded long-range interaction in split-ring-resonator square arrays," Physical Review B: Condensed Matter and Materials Physics, vol. 84, no. 8, Article ID 085416, 2011.

[11] F. B. P. Niesler, N. Feth, S. Linden, and M. Wegener, "Secondharmonic optical spectroscopy on split-ring-resonator arrays," Optics Letters, vol. 36, no. 9, pp. 1533-1535, 2011.

[12] S. Yan and G. A. E. Vandenbosch, "Compact circular polarizer based on chiral twisted double split-ring resonator," Applied Physics Letters, vol. 102, no. 10, Article ID 103503, 2013.

[13] B. Ni, X. S. Chen, L. J. Huang, J. Y. Ding, G. H. Li, and W. Lu, "A dual-band polarization insensitive metamaterial absorber with split ring resonator," Optical and Quantum Electronics, vol. 45, no. 7, pp. 747-753, 2013.

[14] J. Wallauer and M. Walther, "Fano line shape and phase reversal in a split-ring resonator based metamaterial," Physical Review B: Condensed Matter and Materials Physics, vol. 88, no. 19, Article ID 195118, 2013.

[15] Y. Q. Xu, P. H. Zhou, H. B. Zhang, L. Chen, and L. Jiang Deng, "A wide-angle planar metamaterial absorber based on split ring resonator coupling," Journal of Applied Physics, vol. 110, no. 4, Article ID 044102, 2011.

[16] W. J. Padilla, M. T. Aronsson, C. Highstrete, M. Lee, A. J. Taylor, and R. D. Averitt, "Electrically resonant terahertz metamaterials: theoretical and experimental investigations," Physical Review B, vol. 75, no. 4, Article ID 041102, 2007.

[17] C. Imhof and R. Zengerle, "Pairs of metallic crosses as a lefthanded metamaterial with improved polarization properties," Optics Express, vol. 14, no. 18, pp. 8257-8262, 2006.

[18] O. Paul, C. Imhof, B. Reinhard, R. Zengerle, and R. Beigang, "Negative index bulk metamaterial at terahertz frequencies," Optics Express, vol. 16, no. 9, pp. 6736-6744, 2008.

[19] H.-T. Chen, J. F. O’Hara, A. J. Taylor et al., "Complementary planar terahertz metamaterials," Optics Express, vol. 15, no. 3, pp. 1084-1095, 2007.

[20] N. I. Landy, C. M. Bingham, T. Tyler, N. Jokerst, D. R. Smith, and W. J. Padilla, "Design, theory, and measurement of a polarization-insensitive absorber for terahertz imaging," Physical Review B: Condensed Matter and Materials Physics, vol. 79, no. 12, Article ID 125104, 2009.

[21] H. Zhou, F. Ding, Y. Jin, and S. L. He, “Terahertz metamaterial modulators based on absorption," Progress in Electromagnetics Research, vol. 119, pp. 449-460, 2011.

[22] X. Zhou, H. Li, Z. Liu, S. Xie, H. Xu, and X. Peng, "Enhanced optical transmission of non-coaxial double-layer gold nano-slit with slanted sidewall arrays," Solid State Communications, vol. 152, no. 5, pp. 417-421, 2012.

[23] S. Zeng, D. Baillargeat, H.-P. Ho, and K.-T. Yong, "Nanomaterials enhanced surface plasmon resonance for biological and chemical sensing applications," Chemical Society Reviews, vol. 43, no. 10, pp. 3426-3452, 2014. 
[24] E. C. Dreaden, A. M. Alkilany, X. Huang, C. J. Murphy, and M. A. El-Sayed, "The golden age: gold nanoparticles for biomedicine," Chemical Society Reviews, vol. 41, no. 7, pp. 27402779, 2012.

[25] J. Lee, B. Hua, S. Park et al., “Tailoring surface plasmons of highdensity gold nanostar assemblies on metal films for surfaceenhanced Raman spectroscopy," Nanoscale, vol. 6, no. 1, pp. 616623, 2014.

[26] J. M. Luther and J. L. Blackburn, "Optoelectronics: plasmonenhanced plastic devices," Nature Photonics, vol. 7, no. 9, pp. 675-677, 2013.

[27] W. Zhang, W. Ye, C. Wang, W. Li, Z. Yue, and G. Liu, "Silver nanoparticle arrays enhanced spectral surface plasmon resonance optical sensor," Micro \& Nano Letters, vol. 9, no. 9, pp. 585-587, 2014.

[28] D. C. Marinica, A. K. Kazansky, P. Nordlander, J. Aizpurua, and A. G. Borisov, "Quantum plasmonics: nonlinear effects in the field enhancement of a plasmonic nanoparticle dimer," Nano Letters, vol. 12, no. 3, pp. 1333-1339, 2012.

[29] Z.-J. Yang, Z.-S. Zhang, W. Zhang, Z.-H. Hao, and Q.-Q. Wang, "Twinned Fano interferences induced by hybridized plasmons in Au-Ag nanorod heterodimers," Applied Physics Letters, vol. 96, no. 13, Article ID 131113, 2010.

[30] S. Toroghi and P. G. Kik, "Photothermal response enhancement in heterogeneous plasmon-resonant nanoparticle trimers," Physical Review B, vol. 90, no. 20, Article ID 205414, 2014.

[31] P. Drude, "Zur elektronentheorie der metalle," Annalen der Physik, vol. 306, no. 3, pp. 566-613, 1900.

[32] J. Park, H. Kim, and B. Lee, "High order plasmonic Bragg reflection in the metal-insulator-metal waveguide Bragg grating," Optics Express, vol. 16, no. 1, pp. 413-425, 2008.

[33] E. D. Palik, Handbook of Optical Constants and Solids, Academic Press, New York, NY, USA, 1985.

[34] P. B. Johnson and R. W. Christy, "Optical constants of the noble metals," Physical Review B, vol. 6, no. 12, pp. 4370-4379, 1972.

[35] A. Taflove and S. C. Hagness, Computational Electrodynamics: The Finite Difference Time-Domain Method, Artech House, Boston, Mass, USA, 2nd edition, 2000.

[36] J. D. Baena, R. Marqués, F. Medina, and J. Martel, "Artificial magnetic metamaterial design by using spiral resonators," Physical Review B, vol. 69, no. 1, Article ID 014402, 2004.

[37] C.-Y. Chen, I.-W. Un, N.-H. Tai, and T.-J. Yen, "Asymmetric coupling between subradiant and superradiant plasmonic resonances and its enhanced sensing performance," Optics Express, vol. 17, no. 17, pp. 15372-15380, 2009.

[38] Q. Li, B. Zhang, W. Xiong, and J. Shen, "Modulation of the resonance frequency in double-split ring terahertz metamaterials," Optics Communications, vol. 323, pp. 162-166, 2014.

[39] M. Shamonin, E. Shamonina, V. Kalinin, and L. Solymar, "Properties of a metamaterial element: analytical solutions and numerical simulations for a singly split double ring," Journal of Applied Physics, vol. 95, no. 7, pp. 3778-3784, 2004.

[40] K. Aydin, I. Bulu, K. Guven, M. Kafesaki, C. M. Soukoulis, and E. Ozbay, "Investigation of magnetic resonances for different split-ring resonator parameters and designs," New Journal of Physics, vol. 7, no. 1, article 168, 2005. 

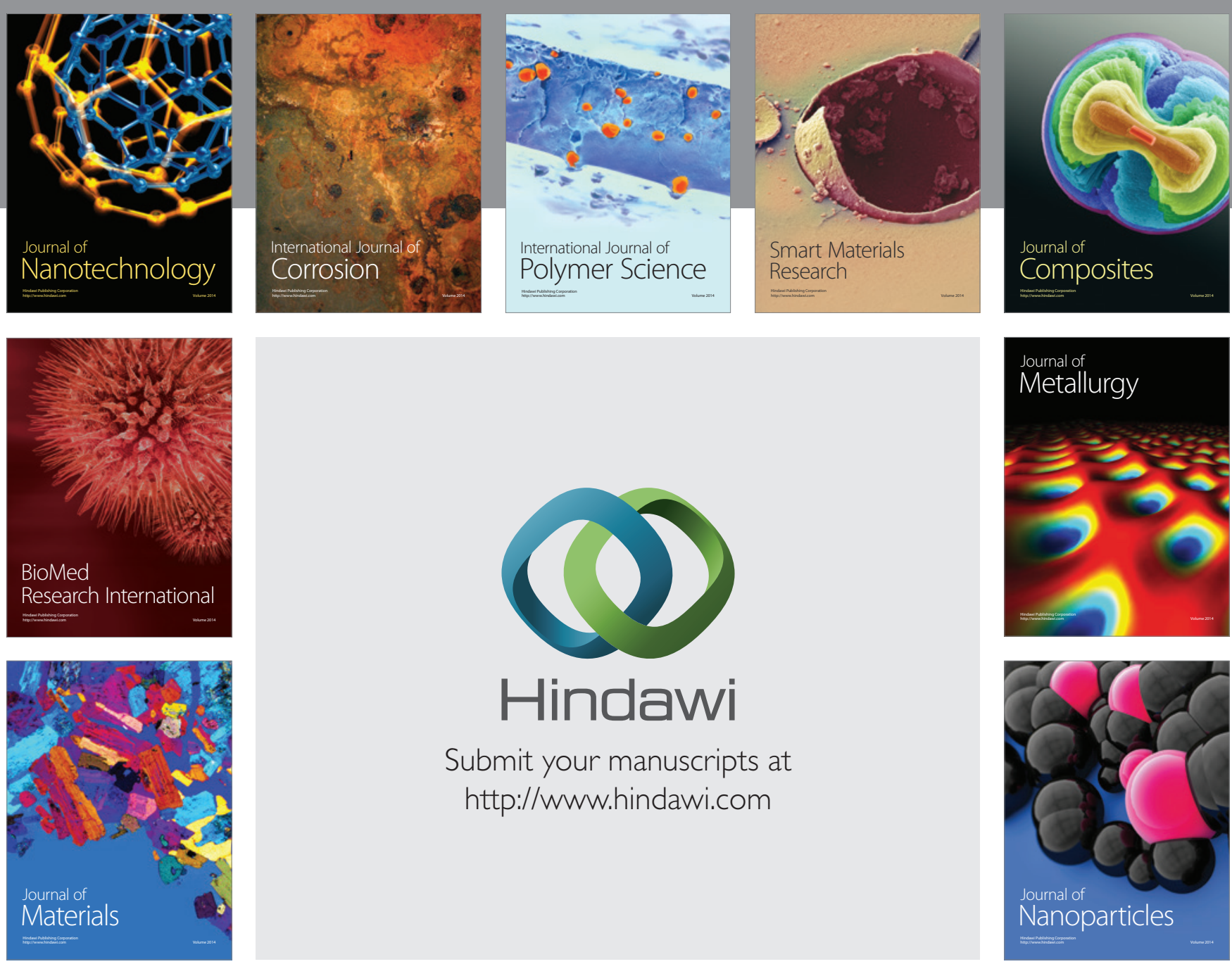

Submit your manuscripts at http://www.hindawi.com
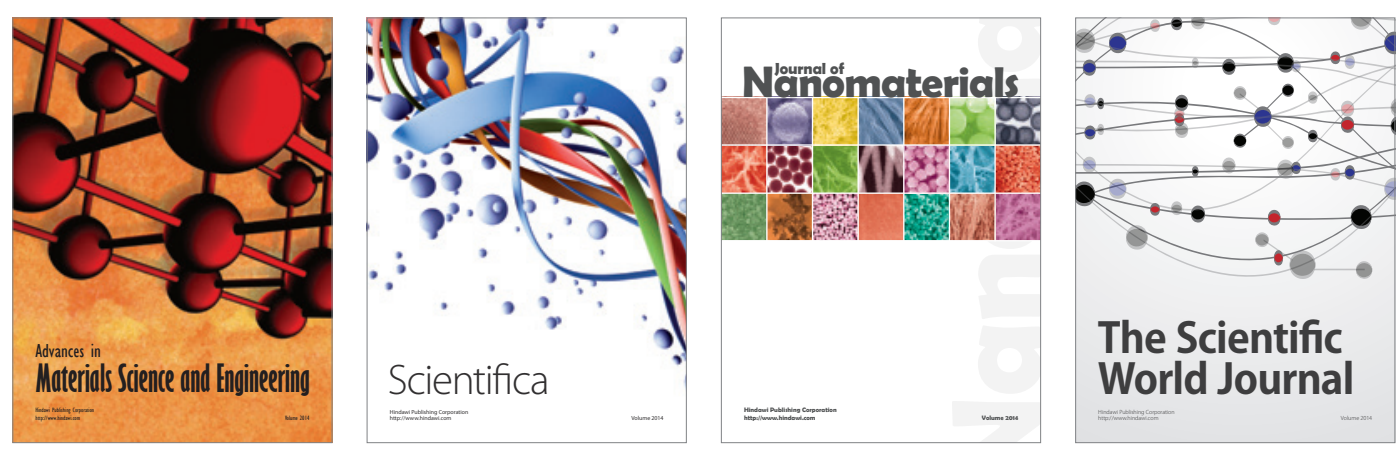

\section{The Scientific World Journal}
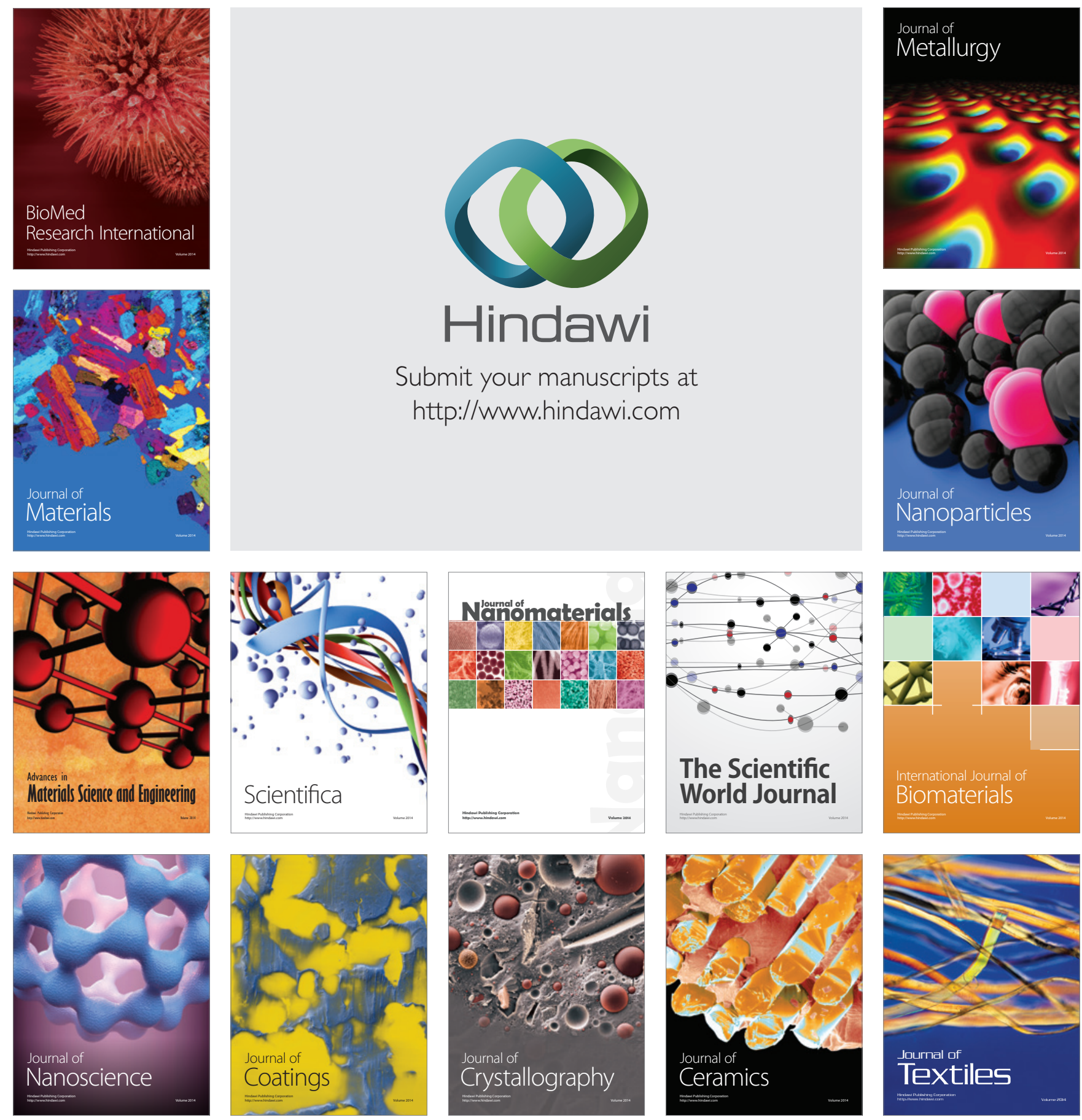